\title{
LETTERS
}

\section{Assessment of asthma control by children and parents}

\section{To the Editor:}

Recent guidelines place a strong emphasis on monitoring asthma control and adjusting treatment accordingly [1]. Several tools are available to assess asthma control, including the asthma control test and childhood asthma control test (C-ACT). These tests were developed to detect uncontrolled asthma and are widely used in research and patient care $[2,3]$.

The C-ACT is a seven-item questionnaire which is designed and validated for children from 4-11 yrs of age and addresses the previous 4 weeks. The C-ACT is divided into two parts. The first is filled in by the child and consists of four questions on perception of asthma control, limitations of activities, coughing and nocturnal awakenings. Response levels vary from 0 to 3 . The second part is filled in by a parent or caregiver and consists of three questions on daytime complaints, daytime wheezing and awakenings at night, with response levels between 0 and 5 . The $\mathrm{C}$-ACT score is the sum of all scores, ranging from 0 (poorest asthma control) to 27 (optimal asthma control). A cut-off of $\leqslant 19$ indicates uncontrolled asthma [4]. Because the C-ACT consists of questions answered by the child and by the parent, this instrument offers the possibility to compare patient's and parents perception on asthma control measured at the same time.

Earlier studies have shown that parents tend to under report asthma symptoms of their child and that children may adapt their activity level to their asthma symptoms $[5,6]$. We hypothesised that parents would underestimate their children's asthma control, and compared C-ACT subscores of children and parents.

We included children aged between 4 and 11 yrs with a doctor's diagnosis of asthma who visited KinderHaven, our outpatient asthma clinic (Rotterdam, the Netherlands), between January and December 2011. All children and parents completed the CACT as part of routine patient care. The child completed the first four questions under supervision of the parent, and then one of the parents completed the remaining questions and calculated the total score before visiting the doctor or nurse. For this study formal medical ethical permission was not required according to the Medical Ethical Committee of Erasmus Medical Centre, Rotterdam, the Netherlands.

To compare asthma control scores between children and parents, Spearman's correlations and the Intraclass Correlation Coefficient (ICC) were calculated. Scores of the children and the parents were expressed as percentage of the maximum, and pairwise compared using the Wilcoxon Signed Ranks Test. To test the effect of age, mean differences per age category were compared using ANOVA. Data were analysed using SPSS (version 17.0; SPSS Inc., Chicago, IL, USA) and a p-value of $<5 \%$ was considered statistically significant.

283 children (mean age 7.7 yrs (range $4-12$ yrs), 66.8\% boys) and their parents completed a C-ACT; 272 C-ACT's were filled in correctly by both child and parent. The median C-ACT score was 22 (interquartile range (IQR) 18-25).

Children scored median $75 \%$ (IQR 58-83\%) of the maximal score, versus $87 \%$ (IQR $73-100 \%$ ) for the parents. On average, children scored $8.3 \%$ lower than their parents (95\% CI 6.4-10.2, $\mathrm{p}<0.001$ ). The upper and lower $95 \%$ limits of agreement were $-40 \%$ and $23 \%$, respectively. The differences between the scores of the child and parent tended to be higher at lower mean scores. One question of the C-ACT is identical for child and parents, the question on nocturnal complaints. For this question children had a median score of $67 \%$ (IQR $67-100 \%$ ), compared to $100 \%$ for parents (IQR $80-100 \%, \mathrm{p}<0.001$ ).

Spearman's correlation between C-ACT-scores of children and parents was $0.72(p<0.001)$ and the ICC was $0.77(p<0.001)$. The difference in scores between children and parents was independent of the child's age $(\mathrm{p}=0.8)$.

We showed that children reported significantly lower asthma control than their parents using the C-ACT. This confirms our hypothesis that parents underestimate the asthma symptoms of their children [6-8]. We speculate that the systematic difference between parents and children might be one of the factors responsible for under treatment of asthma in children [7]. Alternatively, it might also be possible that children overestimate their complaints, and the interpretation of the parent is more accurate. In the absence of any objective gold standard test of asthma control it is impossible to draw conclusions.

In favour of a more accurate perception by the parents is that perception of children may be limited to a shorter recall time, as the common recall period for a child below the age of 8 yrs is only a few days [9], whereas the C-ACT asks about the previous 4 weeks. Parents might be better able to recall symptoms for a longer period of time. However, we found that the difference between children and parents was independent of the child's age, and this argues against this mechanism.

Another reason for the discrepancy between children and parents might be that the questions to be answered by the parents were more quantitative (number of days with complaints) while the child questions were qualitative (how do you feel). In younger children, this may lead to more extreme scores as they will have more trouble in interpreting a neutral state [4]. Comparison between scores is hampered by the fact that most questions to the children and the parents were different. For the single identical question on nocturnal complaints we found children scoring significantly lower than their parents.

We conclude that there was a systematic difference in C-ACT scores by parents and children, with parents tending to under report or children to over report. We recommend considering the scores by parents and children separately when assessing asthma control. 
Sandra Voorend-van Bergen*, Hein Brackel", Daan Caudri*, Johan de Jongste* and Mariëlle Pijnenburg*

*Dept of Paediatric Pulmonolgy, Erasmus MC - Sophia Children's Hospital, Rotterdam, and "Dept of Paediatrics, Catharina Hospital, Eindhoven, The Netherlands.

Correspondence: M. Pijnenburg, Dept of Paediatric Pulmonolgy, Erasmus MC - Sophia Children's Hospital, Dr Molewaterplein 60, 3015 GJ, Rotterdam, the Netherlands. E-mail: m.pijnenburg@erasmusmc.nl

Statement of Interest: None declared.

\section{REFERENCES}

1 Global Initiative for Asthma. Global Strategy for Asthma Management and Prevention. Updated 2011. www.ginasthma.org/ uploads/users/files/GINA_Report2011_May4.pdf

2 Juniper EF, O'Byrne PM, Guyatt GH, et al. Development and validation of a questionnaire to measure asthma control. Eur Respir J 1999; 14: 902-907.
3 Schatz M, Sorkness CA, Li JT, et al. Asthma Control Test: reliability, validity, and responsiveness in patients not previously followed by asthma specialists. J Allergy Clin Immunol 2006; 117: 549-556.

4 Liu AH, Zeiger R, Sorkness C, et al. Development and crosssectional validation of the Childhood Asthma Control Test. J Allergy Clin Immunol 2007; 119: 817-825.

5 Lara M, Duan N, Sherbourne C, et al. Differences between child and parent reports of symptoms among Latino children with asthma. Pediatrics 1998; 102: E68.

6 Davis KJ, Disantostefano R, Peden DB. Is Johnny wheezing? Parentchild agreement in the Childhood Asthma in America survey. Pediatr Allergy Immunol 2011; 22: 31-35.

7 Carroll WD, Wildhaber J, Brand PL. Parent misperception of control in childhood/adolescent asthma: the Room to Breathe survey. Eur Respir J 2012; 39: 90-96.

8 Kieckhefer GM, Lentz MJ, Tsai SY, et al. Parent-child agreement in report of nighttime respiratory symptoms and sleep disruptions and quality. J Pediatr Health Care 2009; 23: 315-326.

9 Eiser C, Morse R. A review of measures of quality of life for children with chronic illness. Arch Dis Child 2001; 84: 205-211.

\section{Chronic beryllium disease: azathioprine as a possible alternative to corticosteroid treatment}

\section{To the Editor:}

Chronic beryllium disease (CBD) is a chronic granulomatous disease that mainly affects the lungs. It occurs after beryllium exposure in genetically susceptible individuals with, most commonly, the HLA-DP $\beta 1$ (Glu69) polymorphism [1]. Beryllium particles are slowly washed out, causing delayed onsets of the disease and flare ups long after exposure to beryllium [2, 3].

The clinical, radiological and pathological presentation of CBD is very similar to sarcoidosis. Thus, misdiagnosis is not uncommon, as reported by FIREMAN et al. [4] and MÜLLER-QUERNHEIM et al. [5]. These authors managed to correct the diagnosis of chronic sarcoidosis to CBD in $4-6 \%$ of patients, thanks to a careful retrospective screening for beryllium exposure [4,5].

In $\mathrm{CBD}$, lungs are damaged by diffuse noncaseating granulomas and this may lead to fibrosis. The US Beryllium Case Registry determined the following specific criteria for CBD diagnosis: a beryllium exposure history; relevant clinical and radiological signs (breathlessness, reduced pulmonary capacity and diffuse interstitial opacities); evidence of beryllium sensitisation with positive beryllium lymphocyte proliferation test in blood or in bronchoalveolar lavage (BAL); and histopathological features such as noncaseating granulomas or mononuclear tissue infiltration without any infection. Long-term prognosis of $\mathrm{CBD}$ is poor with a mortality rate ranging from $5.8 \%$ to $38 \%$. Current treatment of CBD relies on both beryllium exposure cessation and corticosteroid therapy. Corticosteroid therapy is efficient on the granulomatous component and leads to a significant clinical, radiological, biological and functional improvement [6]. However, the efficacy of corticosteroids doesn't last: relapses occur following cessation of therapy or dose lowering and long-duration treatment with possible severe side-effects is often necessary. Eventually, some patients might not respond as expected to corticosteroids, or develop progressive lung fibrosis despite this treatment $[6,7]$.

Herein, we report the first case of CBD successfully treated by azathioprine in a patient for whom corticosteroids were contraindicated.

A 43-yr-old nonsmoking male who had been exposed to beryllium for at least 3 yrs between 1992 and 1997 during his employment as a foundry worker presented to our clinic (Avicenne University Hospital, Bobigny, France). Diffuse interstitial opacities and lymphadenopathy were discovered on chest radiography in 1997. However, it was only in 2001 that CBD diagnosis was considered due to the occurrence of dyspnoea on exertion. Chest computed tomography (CT) evidenced diffuse ground-glass opacities, micronodules and discreet fibrotic lesions in the upper lobes (linear retractile opacities and traction bronchiectasis). A beryllium lymphocytes proliferation test was positive in both blood and BAL (biological index 13.43 and a beryllium statistical index $>2.53$ ). Pulmonary function tests (PFT) showed a restrictive impairment with a $40 \%$ lung volume reduction associated to a decrease of the diffusing capacity of the lung for carbon monoxide (DL,CO) (fig. 1). The serum angiotensine converting enzyme (SACE) level was $70 \mathrm{UI}$ (normal $<40 \mathrm{UI})$. The BAL fluid cell count showed a significant hypercellularity $\left(850,000\right.$ cells $\left.\cdot \mathrm{mL}^{-1}\right)$ and hyperlymphocytosis $(66 \%)$. Genetically, the patient was Glu69 homozygous. There was no granuloma on bronchial biopsy but noncaseating 